\title{
THE ZELEZNIKOW PROBLEM ON A CLASS OF ADDITIVELY IDEMPOTENT SEMIRINGS
}

\author{
YONG SHAO, SINIŠA CRVENKOVIĆ and MELANIJA MITROVIĆ凶
}

(Received 17 January 2012; accepted 11 June 2013; first published online 5 September 2013)

\author{
Communicated by B. Gardner
}

\begin{abstract}
A semiring is a set $S$ with two binary operations + and $\cdot$ such that both the additive reduct $S_{+}$and the multiplicative reduct $S$ • are semigroups which satisfy the distributive laws. If $R$ is a ring, then, following Chaptal ['Anneaux dont le demi-groupe multiplicatif est inverse', C. R. Acad. Sci. Paris Ser. A-B 262 (1966), 274-277], $R_{\bullet}$ is a union of groups if and only if $R_{\bullet}$ is an inverse semigroup if and only if $R_{\bullet}$ is a Clifford semigroup. In Zeleznikow ['Regular semirings', Semigroup Forum 23 (1981), 119-136], it is proved that if $R$ is a regular ring then $R_{\bullet}$ is orthodox if and only if $R_{\bullet}$ is a union of groups if and only if $R_{\bullet}$ is an inverse semigroup if and only if $R_{\bullet}$ is a Clifford semigroup. The latter result, also known as Zeleznikow's theorem, does not hold in general even for semirings $S$ with $S_{+}$a semilattice Zeleznikow ['Regular semirings', Semigroup Forum 23 (1981), 119-136]. The Zeleznikow problem on a certain class of semirings involves finding condition(s) such that Zeleznikow's theorem holds on that class. The main objective of this paper is to solve the Zeleznikow problem for those semirings $S$ for which $S_{+}$is a semilattice.
\end{abstract}

2010 Mathematics subject classification: primary 16Y60, 06F05; secondary 20M18, $20 \mathrm{M} 19$.

Keywords and phrases: semiring, amenable order, regular ordered semigroup, orthodox semigroup, inverse semigroup, Clifford semigroup.

\section{Introduction}

The history of semirings dates back at least to Vandiver [21]. The intensive study of semirings was initiated during the late 1960s when significant applications were found for them. More on applications of semiring theory within analysis, fuzzy set theory, the theory of discrete-event dynamical systems, automata and formal language theory can be found in [8-10]. Thus, semirings now have both a developed algebraic theory as well as important practical applications.

The first author was supported by the Science Foundation of Shaanxi Province, Grant 2011JQ1017. The second author was supported by the Ministry of Education, Science and Technological Development of Serbia, Grant 174108. The third author was supported by the Ministry of Education, Science and Technological Development of Serbia, Grant 174026.

(C) 2013 Australian Mathematical Publishing Association Inc. 1446-7887/2013 \$16.00 
A semiring $(S,+, \cdot)$ is a set $S$ equipped with two binary operations + and $\cdot$ such that the following identities are satisfied:

(SR1) $\quad x+(y+z) \approx(x+y)+z$

(SR2) $\quad x(y z) \approx(x y) z$

(SR3) $x(y+z) \approx x y+x z,(x+y) z=x z+y z$.

A semigroup $S_{+}=(S,+)$ is called the additive reduct, and a semigroup $S \bullet=(S, \cdot)$ is called the multiplicative reduct of a semiring $S$. Let $\mathcal{P}$ be a class of semigroups. A semiring $S$ is an additively $\mathcal{P}$ semiring, or $a-\mathcal{P}$ semiring for short, if $S_{+}$is a semigroup from class $\mathcal{P}$. In a similar manner we can define a multiplicative $\mathcal{P}$ semiring, or $m-\mathcal{P}$ semiring for short. If both $S_{+}$and $S$. are from class $\mathcal{P}$, then $S$ is a $\mathcal{P}$ semiring. For example, a semiring $S$ is $m$-regular if for any $a \in S$ there exists $x \in S$ such that $a=$ axa.

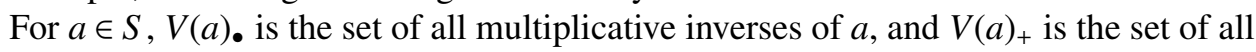
additive inverses of $a$. If these sets are nonempty then we can distinguish the following three subsets of a semiring $S$ : the set $E(S)_{\bullet}$ of all multiplicative idempotents of $S \bullet$; the set $E(S)_{+}$of all additive idempotents of $S_{+}$; and $E(S)=E(S)_{\bullet} \cap E(S)_{+}$. An orthodox semiring is an $m$-regular semiring $S$ with a subsemigroup $E(S)$ • of $S$.. A semiring $S$ is additively idempotent if $S=E(S)_{+}$, and $S$ is idempotent if $S=E(S)$.

Let us briefly recall that if $R$ is a ring, then, following [6], $R_{\bullet}$ is a union of groups if and only if $R_{\bullet}$ is an inverse semigroup if and only if $R_{\bullet}$ is a Clifford semigroup. In [23], it is proved that if $R$ is a regular ring then $R_{\bullet}$ is orthodox if and only if $R_{\bullet}$ is a union of groups if and only if $R_{\bullet}$ is an inverse semigroup if and only if $R_{\bullet}$ is a Clifford semigroup. The latter result, also known as Zeleznikow's theorem, does not hold for an arbitrary semiring $[22,23]$. The Zeleznikow problem on a certain class of semirings involves finding condition(s) such that Zeleznikow's theorem holds on that class. In the literature concerned with $(a-, m$-)inverse semirings, (with $a$-commutativity or not, and/or with zero, and/or with the identity), the solution of the Zeleznikow problem is mostly described in terms of its (special) elements [20]. The aim of this paper is to make a little progress in that direction. The following example, taken from [23], shows that Zeleznikow's theorem does not hold even for semirings $S$ with $S_{+}$a semilattice.

Example. Let $(S,+)$ be a semilattice with $|S| \geq 2$. Let us define $x \cdot y=x$ for all $x, y \in S$. Then $(S,+, \cdot)$ is a semiring with $S$. orthodox but not inverse.

This motivates us to try to solve the Zeleznikow problem for those semirings $S$ for which $S_{+}$is a semilattice. In order to achieve this, a study of natural partial orders $\leq_{+}$ and $\leq_{\text {. }}$ of such semirings will be very helpful. In what follows, (one- and two-sided) amenability properties, taken from semigroup theory, and used here in semiring theory for the first time, will be of help. Due to their natural and close connections, many ideas, results and methods of semigroup theory find their place and become important within the semiring theory, too.

The paper is organized in the following way. Section 2 gives a short history of the Zeleznikow problem, as well as the semigroup theory background needed for our main objective_-proving Zeleznikow's theorem for semirings $S$ with $S_{+}$a semilattice. Inverse semirings $S$ with the property that $\leq_{+}$extends $\leq_{\bullet}$ on $S$ and its influence to 
certain subsets of $S$ are considered in Section 3. The main result of this section, Proposition 3.2, gives conditions under which $\leq_{+}$extends $\leq_{\text {. }}$ on the semirings in question. In Section 4 we move a little further with our constraints on natural partial orders on $S$. Concepts of one- and two-sided amenability taken from semigroup theory (and applied on $\leq_{+}$) are defined and adopted for the semiring case. It is shown that $\leq_{+}$ need not, in general, be amenable even when the semiring in question is a Clifford semiring. The main result of this section, indeed of the entire paper, is Theorem 4.6, in which Zeleznikow's theorem for semirings $S$ with $S_{+}$a semilattice is proved. We end this section (and the paper) with the criteria (Theorem 4.8) for a Clifford semiring that guarantee amenability of $\leq_{+}$.

We refer to [7] as a source of references on semirings. For notation and terminology not given in this paper we refer to $[11,17,18]$ as background on semigroup theory, and [2] as background on ordered algebraic structure theory.

\section{History and background}

This section gives a short history of the Zeleznikow problem, as well as the semigroup theory background needed for proving our main objective-Zeleznikow's theorem for semirings $S$ with a semilattice $S_{+}$.

2.1. History of the Zeleznikow problem. The story began with Chaptal who in 1966 proved the following result [6, Proposition 1].

Proposition 2.1. For a ring $(R,+, \cdot)$ the following conditions are equivalent:

(i) $R_{\bullet}$ is a union of groups;

(ii) $R_{\bullet}$ is an inverse semigroup;

(iii) $R_{\bullet}$ is a Clifford semigroup.

In 1981, Zeleznikow [23] gave the next theorem, now known as Zeleznikow's theorem.

THEOREM 2.2. For a regular ring $R$ the following conditions are equivalent:

(i) $R_{\bullet}$ is an orthodox semigroup;

(ii) $R_{\bullet}$ is a union of groups;

(iii) $R_{\bullet}$ is an inverse semigroup;

(iv) $R_{\bullet}$ is a Clifford semigroup.

In [22] the ring-semigroup, that is, the semigroup $(S, \cdot)$ for which there exists a binary operation $+: S \times S \longrightarrow S$ such that $(S,+, \cdot)$ is a ring, is defined. Zeleznikow's theorem for a regular ring-semigroup [22, Theorem 13] is as follows.

THEOREM 2.3. For a regular ring-semigroup $(S, \cdot)$ the following are equivalent:

(i) $(S, \cdot)$ is orthodox;

(ii) $(\forall e, f \in E(S)) e f=0 \Rightarrow f e=0$;

(iii) $(S, \cdot)$ is inverse;

(iv) $(S, \cdot)$ is Clifford. 
Zeleznikow has shown that for additively inverse semirings, even for a semilattice $S_{+}$, we do not have a solution of the Zeleznikow problem (see the example given above). Sen and Maity in 2004 gave the following result [20, Theorem 2.8].

THEOREM 2.4. Let $(S,+, \cdot)$ be an a-commutative and a-inverse semiring satisfying

(A) $a\left(b+b^{\prime}\right)=\left(b+b^{\prime}\right) a$,

(B) $a+a\left(b+b^{\prime}\right)=a$,

for any $a, b \in S$, where $a^{\prime} \in V(a)_{+}, b^{\prime} \in V(b)_{+}$. If, in addition, $S$. is a regular semigroup, then the following conditions are equivalent:

(i) $S_{\bullet}$ is an orthodox semigroup;

(ii) $S \bullet$ is a union of groups;

(iii) $S_{\bullet}$ is an inverse semigroup;

(iv) $S$. is a Clifford semigroup.

2.2. Main objective. Our main objective in this paper is to prove Zeleznikow's theorem for semirings $S$ with a semilattice $S_{+}$.

Theorem 2.5. Let $(S,+, \cdot)$ be a semiring with $S_{+}$a semilattice and $S$. a regular semigroup. If $\leq_{+} i$ an amenable order on $S$ then the following conditions are equivalent:

(i) $S_{.}$is an orthodox semigroup;

(ii) $S_{.}$is a union of groups;

(iii) $S_{\bullet}$ is an inverse semigroup;

(iv) $S_{\bullet}$ is a Clifford semigroup.

2.3. Semigroup theory background. In order to achieve our main objective, a study of the natural orders $\leq_{+}$and $\leq_{\text {. }}$ of semirings in question will be very helpful. In what follows, (one- and two-sided) amenability properties, taken from semigroup theory, and here used in semiring theory for the first time, will be of help. Due to their natural and close connections, many ideas, results and methods of semigroup theory find their place and become important within the semiring theory, too.

A partial order on a semigroup $S$ is called natural if it is defined by means of the multiplication on $S$. For our purpose here, we recall that the natural partial order $\leq_{n}$ on a regular semigroup is given by

$$
a \leq_{n} b \Leftrightarrow(\exists e, f \in E(S)) \quad a=e b=b f
$$

A useful property of the natural partial order is compatibility with multiplication, that is, $a \leq_{n} b$ and $c \in S$ implies $a c \leq_{n} b c$ and $c a \leq_{n} c b$. But, already for regular semigroups, compatibility does not hold in general. It is also known that for a regular semigroup $S$, natural partial order is compatible with multiplication if and only if $S$ is locally inverse. Locally inverse or pseudo inverse semigroups include classes of generalized inverse or orthodox locally inverse semigroups (that is, regular semigroups with $E(S)$ a normal band) and inverse semigroups. 
An inverse semigroup is a semigroup $S$ such that for any $a \in S$ there is unique element, denoted by $a^{-1}$, such that $a=a a^{-1} a$ and $a^{-1}=a^{-1} a a^{-1}$. Natural partial order for such semigroups, defined as

$$
a \leq_{n} b \Leftrightarrow(\exists e \in E(S)) \quad a=b e,
$$

is an important ingredient in their structure. We refer the reader to [17] for a background material on inverse semigroups and their natural partial orders. Besides the fact that almost all the results concerning orderings on an inverse semigroup have focused on the natural partial order, notable exceptions appear as well. For example, the concept of one and two-sided amenability appears in the study of compatible orders definable on an inverse semigroup. We recall from [12] that a partial order $\leq$ defined on an inverse semigroup $S$ is left amenable if it is compatible with multiplication on $S$, extends the natural partial order $\leq_{n}$ on idempotents and is such that

$$
a \leq b \Rightarrow a^{-1} a \leq b^{-1} b .
$$

A right amenable order is defined dually, while an amenable order is one which is both left and right amenable. Clearly, any inverse semigroup is amenably ordered under $\leq_{n}$. Amenably ordered inverse semigroups have been considered within investigations into Dubreil-Jacotin regular semigroups in [1, 13, 14]. Left amenable ordered inverse senigroups are considered in detail in [12]. It is also shown in [12] that (two-sided) amenability 'imposes strong structural constraints on the structure of ordered inverse semigroups'. That is why a study of the one-sided nature of these orders has been initiated in a more general setting [3-5]. Here, in what follows, our objective is to obtain a significant generalization of the case where $S$ is a certain kind of semiring.

\section{On inverse semirings with $\leq . \subseteq \leq_{+}$}

Throughout this paper we will consider semirings with some additional properties. Our starting point is a semiring $(S,+, \cdot)$ with idempotent and commutative addition:

(SR4) $x+x \approx x$

(SR5) $x+y \approx y+x$

that is, a semiring $S$ with a semilattice $S_{+}$. For such a semiring $S$ we have that $S=E(S)_{+}$, so $E(S)=E(S)_{+} \cap E(S)_{\bullet}=E(S)_{\bullet}$. Clearly, $S$ is regular (completely regular, inverse or Clifford) if $S_{\bullet}$ is regular (completely regular, inverse or Clifford). A semiring $S$ is a bisemilattice if $S$. is also a semilattice. A distributive lattice is a bisemilattice which satisfies the absorption law $x+x y \approx x$ and $x(x+y) \approx x$. A monobisemilattice is a bisemilattice which satisfies $x+y \approx x y$.

In what follows, a useful tool will be natural partial orders $\leq_{+}$and $\leq_{\text {• }}$ defined on $S_{+}$ and $S$. respectively by

$$
\begin{aligned}
& a \leq_{+} b \Leftrightarrow a+b=b+a=b, \\
& a \leq_{\bullet} b \Leftrightarrow\left(\exists e, f \in E(S)_{\bullet}\right) a=b e=f b,
\end{aligned}
$$


$a, b \in S$. If $S$ is an inverse semiring then $\leq_{+}$is compatible with both operations

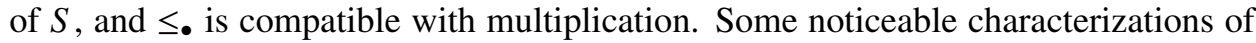
semirings (especially from a certain subclass of idempotent semirings) are given in terms of natural partial orders and their relationships. For example, in [19], the membership in certain subvarieties of the variety of idempotent semirings is given using the relation $\leq_{+} \subseteq \leq_{\text {. }}$. In [16], the variety of all idempotent semirings having Green's $\mathcal{D}$ relation as congruence is described using the connection $\leq_{+} \subseteq \geq_{\text {• }}$ and the equality $\leq_{+}=\geq_{\text {. }}$. In this section we consider an inverse semiring $S$ with the property that $\leq_{+}$extends $\leq_{\bullet}$, that is,

$$
\leq_{\bullet} \subseteq \leq_{+},
$$

and its influence on certain subsets of $S$. The following result on inverse semirings obtained in [12] will be useful.

THeOREM 3.1. If $S$ is an inverse semiring then $E(S)$ is a bisemilattice.

The main result of this section needed to solve our main problem, which we set in the previous section, gives conditions under which $\leq_{+}$extends $\leq_{\bullet}$ on $S$.

Proposition 3.2. Let $S$ be an inverse semiring. Then, the following conditions are equivalent:

(i) $\leq_{+}$is an extension of $\leq_{\bullet}$ (that is, $\leq_{\bullet} \subseteq \leq_{+}$);

(ii) $(\forall e, f \in E(S)) e \leq_{+} f \Leftrightarrow e \leq_{\bullet} f$;

(iii) $(\forall a, b \in S) a+a b^{-1} b=a$;

(iv) $E(S)$ is a distributive lattice.

Proof. Let $S$ be an inverse semiring.

(i) $\Rightarrow$ (ii). Let $e, f \in E(S)$. If $e \leq_{\bullet} f$, then, by (i), $e \leq_{+} f$ follows immediately. Conversely, if $e \leq_{+} f$, then $e=e^{2} \leq_{+} e f$. From $e f \leq_{\bullet} e(e f=e \cdot e f=e f \cdot f)$ and (i) we have that $e f \leq_{+} e$. Thus $e f=e$ holds, that is, $e \leq_{\bullet} f$, as required.

(ii) $\Rightarrow$ (i). Let $a \leq_{\bullet} b, a, b \in S$. Then, by [11, Proposition 5.2.1], we have that $b^{-1} a=a^{-1} a, a a^{-1} \leq_{\bullet} b b^{-1}$ and $a^{-1} a \leq_{\bullet} b^{-1} b$. Now, by (ii), we get $a^{-1} a \leq_{+} b^{-1} b$, $a a^{-1} \leq_{+} b b^{-1}$. So

$$
\begin{aligned}
& a \leq_{+} b b^{-1} a \quad \text { (post-multiplying } a a^{-1} \leq_{+} b b^{-1} \text { by } a \text { ) } \\
& \leq_{+} b a^{-1} a \quad\left(b^{-1} a=a^{-1} a\right) \\
& \leq_{+} b b^{-1} b \quad\left(a^{-1} a \leq_{+} b^{-1} b\right) \\
& =b \text {. }
\end{aligned}
$$

Thus $\leq_{\bullet} \subseteq \leq_{+}$.

(ii) $\Rightarrow$ (iii). Let $a, b \in S$. Then $a^{-1} a b^{-1} b \leq_{\bullet} a^{-1} a$, and, by (ii), it follows that $a^{-1} a b^{-1} b \leq_{+} a^{-1} a$, that is, $a^{-1} a b^{-1} b+a^{-1} a=a^{-1} a$. Multiplying this by $a$, that is, from $a\left(a^{-1} a b^{-1} b+a^{-1} a\right)=a a^{-1} a$, we obtain $a b^{-1} b+a=a$.

(iii) $\Rightarrow$ (iv). Follows immediately by Theorem 3.1 and (iii).

(iv) $\Rightarrow$ (ii). Let $e, f \in E(S)$ be such that $e \leq_{+} f$, that is, $e+f=f$. Then $e+e f=e f$ and, by the absorption law satisfied in $E(S)$, it follows that $e=e+e f=e f$, that 
is, $e \leq_{\bullet} f$. Conversely, if $e \leq_{\bullet} f$, that is, $e f=e$, then, by the absorption law again, we have $f=e f+f=e+f$. Thus $e \leq_{+} f$.

If $B$ is a bisemilattice then, as is proved [15], $B$ is a distributive lattice if and only if $\leq_{\bullet}=\leq_{+}$. Now we have the following result.

THEOREM 3.3. If $S$ is an inverse semiring satisfying $\leq_{\bullet}=\leq_{+}$then $S$ is a distributive lattice.

Proof. Let $a, b \in S$ be such that $a \mathcal{L}^{\bullet} b$, which, by [11, Theorem 5.1.2], means that $a^{-1} a=b^{-1} b$. From $a \leq_{+} a+b$ and the assumption we have $a \leq_{\bullet} a+b$. Now, by [11, Proposition 5.2.1], we have that $a^{-1} \leq_{\bullet}(a+b)^{-1}$, that is, $a^{-1}=a^{-1} a(a+b)^{-1}$. In a similar manner, from $b \leq_{\bullet} a+b$ we can obtain $b^{-1}=b^{-1} b(a+b)^{-1}$. So

$$
a^{-1}=a^{-1} a(a+b)^{-1}=b^{-1} b(a+b)^{-1}=b^{-1} .
$$

Thus $a=b$ and every $\mathcal{L}^{\bullet}$-class of $S$. has only one element which, by [11, Theorem 5.1.2], means that $E(S)=S$, and, by Proposition 3.2, $S$ is a distributive lattice.

Proposition 3.4. Let $S$ be an inverse semiring. Then, the following conditions are equivalent:

(i) $(\forall e, f \in E(S)) e+f \leq_{\bullet} e$;

(ii) $(\forall a, b \in S) a+a b^{-1} b=a b^{-1} b$;

(iii) $E(S)$ is a monobisemilattice.

Proof. Let $S$ be an inverse semiring.

(i) $\Rightarrow$ (iii). Let $e, f \in E(S)$. Then, by (i), it follows that $e+f \leq_{\bullet} e$ and $e+f \leq_{\bullet} f$, which further imply that $(e+f)^{2} \leq_{\bullet} e f$. By Theorem 3.1, it follows that $(e+f) \leq_{\bullet} e f$, that is,

$$
\begin{aligned}
e+f & =e f(e+f) \\
& =e f e+e f \\
& =e f+e f \\
& =e f,
\end{aligned}
$$

so $E(S)$ is a monobisemilattice.

(iii) $\Rightarrow$ (ii). Let $a, b \in S$. By (iii) we have that $a^{-1} a+b^{-1} b=a^{-1} a b^{-1} b$. Then $a\left(a^{-1} a+b^{-1} b\right)=a a^{-1} a b^{-1} b$ implies that $a+a b^{-1} b=a b^{-1} b$, as required.

(ii) $\Rightarrow$ (i). Let $e, f \in E(S)$. Then, by (ii), $e+e f=e f$ and $f+f e=f e$. Now, by Theorem 3.1,

$$
\begin{aligned}
e+f & =(e+f)^{2} \\
& =e+e f+f e+f \\
& =(e+e f)+(f+f e) \\
& =e f+f e \\
& =e f \\
& \leq e .
\end{aligned}
$$

Thus $e+f \leq_{\bullet} e$. 


\section{On regular semirings with $\leq_{+}$left amenable}

Let $\leq$ be a partial order defined on a regular semiring $S$. Following [3-5], for the semigroup case, we can here extend it to the semiring case and give the following definition.

A partial order $\leq$ defined on a regular semiring $S$ is left amenable if it satisfies the following conditions:

(i) $\leq$ is compatible with the operations of $S$;

(ii) $\leq$ is an extension of the natural partial order $\leq_{\text {: }}$;

(iii) $a \leq b$ implies that there exist an inverse $a^{*} \in V(a)$. and an inverse $b^{*} \in V(b)$. such that $a^{*} a \leq . b^{*} b$.

A right amenable partial order is defined dually. A left and right amenable order $\leq$ is called amenable.

Conditions (ii) and (iii) given above can be replaced by the following.

(la) $\leq$ is a left amenable order on $S_{\text {. }}$.

The importance of condition (i) is illustrated in the next example.

EXAMPLE 4.1. Let $S$ be a three-element bisemilattice with operation tables

\begin{tabular}{c|ccc}
+ & $a$ & $b$ & $c$ \\
\hline$a$ & $a$ & $b$ & $c$ \\
$b$ & $b$ & $b$ & $b$ \\
$c$ & $c$ & $b$ & $c$
\end{tabular}

\begin{tabular}{c|ccc}
$\cdot$ & $a$ & $b$ & $c$ \\
\hline$a$ & $a$ & $a$ & $a$ \\
$b$ & $a$ & $b$ & $b$ \\
$c$ & $a$ & $b$ & $c$
\end{tabular}

The natural partial order $\leq_{\bullet}$ is left amenable on $S_{\bullet}$, but is not compatible with addition in $S$. In fact, $a \leq_{\bullet} b, a+c=c, b+c=b$, but $b \leq_{\bullet} c$.

The next lemma will be useful later.

Lemma 4.1. Let $S$ be a regular semiring and let $\leq$ be an amenable partial order defined on it. Then the following conditions are equivalent:

(i) $\leq$ extends the natural partial order $\leq$.;

(ii) $\leq$ extends $\leq$. on $E(S)$;

(iii) $\leq$ coincides with $\leq$. on $E(S)$.

Proof. (i) $\Rightarrow$ (ii). Obvious.

(ii) $\Rightarrow$ (iii). Let $e \leq f, e, f \in E(S)$. Then, by the assumption, we have that $e e^{*} \leq$. $f f^{*}$ and $e^{\prime} e \leq f^{\prime} f$ for some $e^{*}, e^{\prime} \in V(e)$. and $f^{*}, f^{\prime} \in V(f)_{\text {. }}$. Thus, $e e^{*}=f f^{*} e e^{*}$ and $e^{\prime} e=e^{\prime} e f^{\prime} f$. So, from $e=f f^{*} e e^{*} e=f f^{*} e$ we get $f e=f \cdot f f^{*} e=f f^{*} e=e$. Similarly, from $e^{\prime} e=e^{\prime} e f^{\prime} f$ we have $e=e f$. Therefore $e=e f=f e$, that is, $e \leq_{\bullet} f$, as required.

(iii) $\Rightarrow$ (i). Let $a \leq \bullet b, a, b \in S$. Then, by [2, Theorem 13.6] for any $b^{*} \in V(b)$. there exists $a^{*} \in V(a)$. such that $a=b a^{*} a$ and $a^{*} a \leq b^{*} b$. Now, by (iii), $a^{*} a \leq b^{*} b$, which give $a=b a^{*} a \leq . b b^{*} b=b$ and we have $a \leq b$. 
In the previous section we considered an inverse semiring $S$ with property that $\leq_{+}$ extends $\leq_{\text {. }}$. Here we go a little further with our constraints-we assume that $\leq_{+}$is one- or two-sided amenable. But let us first remark that $\leq_{+}$need not be amenable even when the semiring in question is a Clifford semiring. In order to illustrate this we have the following example.

EXAMPLE 4.2. Let $G=\langle a\rangle$ be a cyclic group with infinite order generated by $a$, that is, $G=\left\{a^{n} \mid n \in Z\right\}$, where $a^{0}=e$ is the identity of $G$. We can adjoin an extra element 1 to $G$, and write $G \cup\{1\}$ as $G^{1}$. If we define the multiplication on $G^{1}$ by

$$
(\forall n \in Z) a^{n} \cdot 1=1 \cdot a^{n}=a^{n} \quad \text { and } \quad 1 \cdot 1=1,
$$

then $G^{1}$ becomes a Clifford semigroup and $E\left(G^{1}\right)=\{e, 1\}$. Define a total order $\leq$ on $G^{1}$ as follows:

$$
\cdots \leq a^{-2} \leq a^{-1} \leq e \leq 1 \leq a \leq a^{2} \leq \cdots .
$$

It is easy to verify that $\leq$ is compatible with multiplication on $G^{1}$. Also, we can define the addition + on $G^{1}$ by $u+v=\max \{u, v\}$ and so $\left(G^{1},+, \cdot\right)$ becomes a Clifford semiring. In fact, on the Clifford semiring $\left(G^{1},+, \cdot\right)$, we have $\leq=\leq_{+}$. If we assume that $\leq_{+}$is left amenable, then $1 \leq_{+} a$ implies $1=1 \cdot 1 \leq_{\bullet} a^{-1} a=e$, which is a contradiction.

In the rest of this paper we will consider semirings with $\leq_{+}$one- or two-sided amenable. In what follows (within this section), we will prove that one- and twosided amenability coincide for inverse semirings. Using that result (and some others), we will give a solution to the Zeleznikow problem for semirings $S$ with a semilattice $S_{+}$.

Some equivalent conditions to the left amenability of $\leq_{+}$on an inverse semiring are given in the following theorem.

Theorem 4.2. Let $S$ be an inverse semiring. Then, the following conditions are equivalent:

(i) $\leq_{+}$is left amenable;

(ii) $E(S)$ is a distributive lattice and

$$
(\forall a, b \in S)(a+b)^{-1}(a+b)=a^{-1} a+b^{-1} b ;
$$

(iii) S satisfies

$$
(\forall a, b \in S) a=a(a+b)^{-1}(a+b)
$$

(iv) $(\forall a, b \in S) a \in S(a+b)$.

Proof. (i) $\Rightarrow$ (ii). By Proposition 3.2 we have that $E(S)$ is a distributive lattice. Let $a, b \in S$. From $a \leq_{+} a+b$ and $b \leq_{+} a+b$, by the assumption, we have that $a^{-1} a \leq$. $(a+b)^{-1}(a+b)$ and $b^{-1} b \leq_{\bullet}(a+b)^{-1}(a+b)$, which implies, by the assumption again, $a^{-1} a \leq_{+}(a+b)^{-1}(a+b)$ and $b^{-1} b \leq_{+}(a+b)^{-1}(a+b)$. So $a^{-1} a+b^{-1} b \leq_{+}$ $(a+b)^{-1}(a+b)$. On the other hand, it is obvious that $a b^{-1} b \leq_{\bullet} a$ and $b a^{-1} a \leq_{\bullet} b$, 
so we get $a b^{-1} b \leq_{+} a$ and $b a^{-1} a \leq_{+} b$. Thus we have that $(a+b)\left(a^{-1} a+b^{-1} b\right)=$ $a+a b^{-1} b+b a^{-1} a+b=a+b$. Now from $(a+b)^{-1}(a+b)\left(a^{-1} a+b^{-1} b\right)=(a+b)^{-1}$ $(a+b)$ it follows that $(a+b)^{-1}(a+b) \leq_{\bullet} a^{-1} a+b^{-1} b$. By Proposition 3.2 we have that $(a+b)^{-1}(a+b) \leq_{+} a^{-1} a+b^{-1} b$. Hence $(a+b)^{-1}(a+b)=a^{-1} a+b^{-1} b$.

(ii) $\Rightarrow$ (i). Let $S$ be an inverse semiring and $E(S)$ a distributive lattice which satisfies the given equality. By Proposition 3.2 we have that $\leq_{\bullet} \subseteq_{+}$. Let $a \leq_{+} b$, that is, $a+b=$ $b$ and $(a+b)^{-1}=b^{-1}$. So $(a+b)^{-1}(a+b)=b^{-1} b$. Now, by the assumption, it follows that $a^{-1} a+b^{-1} b=b^{-1} b$, which implies that $a^{-1} a \leq_{+} b^{-1} b$. By Proposition 3.2 again, we have that $a^{-1} a \leq_{\bullet} b^{-1} b$. Thus, $\leq_{+}$is left amenable.

(i) $\Rightarrow$ (iii). From $a \leq_{+} a+b, a, b \in S$, by the assumption, we have that $a^{-1} a \leq$. $(a+b)^{-1}(a+b)$, that is, $a^{-1} a=a^{-1} a(a+b)^{-1}(a+b)$. So $a=a a^{-1} a=a a^{-1} a(a+b)^{-1}$ $(a+b)=a(a+b)^{-1}(a+b)$, as required.

(iii) $\Rightarrow$ (iv). This is obvious.

(iv) $\Rightarrow$ (i). Let $a \leq_{+} b$, that is, $a+b=b$. Then, by (iv), there exists $x \in S$ such that $a=x(a+b)$. Thus $a^{-1}=(a+b)^{-1} x^{-1}$, and we have $a^{-1} a=(a+b)^{-1} x^{-1} x(a+b)$. It can easily be checked that $(a+b)^{-1} x^{-1} x(a+b) \leq_{\bullet}(a+b)^{-1}(a+b)$, and we have $a^{-1} a \leq_{\bullet}(a+b)^{-1}(a+b)$, that is, $a^{-1} a \leq_{\bullet} b^{-1} b$.

Let $a \leq_{\bullet} b, a, b \in S$. Then, by [11, Proposition 5.2.1], we have that $a^{-1} a \leq_{\bullet} b^{-1} b$, that is, $a^{-1} a=b^{-1} b a^{-1} a$, so

$$
a^{-1} a+b^{-1} b=b^{-1} b a^{-1} a+b^{-1} b=b^{-1} b\left(a^{-1} a+b^{-1} b\right) .
$$

There exists, by (iv), $y \in S$ such that $b^{-1} b=y\left(a^{-1} a+b^{-1} b\right)$. So

$$
b^{-1} b\left(a^{-1} a+b^{-1} b\right)=y\left(a^{-1} a+b^{-1} b\right)=b^{-1} b .
$$

In a similar manner we can obtain $b^{-1} b=\left(a^{-1} a+b^{-1} b\right) b^{-1} b$. Now, by (4.1), we have that $a^{-1} a+b^{-1} b=b^{-1} b$, that is, $a^{-1} a \leq_{+} b^{-1} b$, which implies $b a^{-1} a \leq_{+} b$. By [11, Proposition 5.2.1] again, we have $b a^{-1} a=a a^{-1} a=a \leq_{+} b$. Thus $a \leq_{+} b$, and $\leq_{+}$is an extension of $\leq$.

Some properties of an inverse semiring with $\leq_{+}$left amenable are given in the following theorem.

THEOREM 4.3. Let $S$ be an inverse semiring with $\leq_{+}$left amenable. Let $a, b \in S$ and $e \in E(S)$. Then the following conditions hold:

(i) $a \leq_{+} b$ implies $a=a b^{-1} b$ and $a b^{-1} \leq_{+} a a^{-1}$;

(ii) $a \leq_{+} e$ implies $a^{-1} a \leq_{\bullet} a a^{-1}$ and $a=a^{2} a^{-1}$;

(iii) $a \leq_{+} e$ implies $a^{-1} a=a a^{-1}$.

Proof. (i). Let $a \leq_{+} b$. Then we have $a a^{-1} a b^{-1} \leq_{+} a a^{-1} b b^{-1}$, that is, $a b^{-1} \leq_{+}$ $a a^{-1} b b^{-1}$. On the other hand, we have $a a^{-1} b b^{-1} \leq_{\bullet} a a^{-1}$, which, by the assumption, implies $a a^{-1} b b^{-1} \leq_{+} a a^{-1}$, and we have $a b^{-1} \leq_{+} a a^{-1}$. By Proposition 3.2, we have $a+a b^{-1} b=a$, that is, $a b^{-1} b \leq_{+} a$. On the other hand, by [11, Proposition 5.2.1], we have $a^{-1} a \leq_{+} b^{-1} b$, so $a=a a^{-1} a \leq_{+} a b^{-1} b$. Thus $a=a b^{-1} b$. 
(ii). Let $a \leq_{+} e$. By (i), we have $a=a e$ and $a e \leq_{+} a a^{-1}$, which, further, by left amenability gives $a^{-1} a \leq_{\bullet}\left(a a^{-1}\right)^{-1} a a^{-1}=a a^{-1}$, which implies $a^{-1} a=a^{-1} a^{2} a^{-1}$. So

$$
a=a a^{-1} a=a a^{-1} a^{2} a^{-1}=a a^{-1} a a a^{-1}=a^{2} a^{-1} .
$$

(iii). If $a \leq_{+} e$, then, by (ii), $a^{-1} a \leq_{\bullet} a a^{-1}$ follows. Thus, we have to prove that $a a^{-1} \leq_{\bullet} a^{-1} a$.

It is clear that $a a^{-1} \leq_{+} a a^{-1}+a^{-2} a$. Since $\leq_{+}$is left amenable on the multiplicative reduct of $S$, it follows that $a a^{-1} \leq_{\bullet}\left(a a^{-1}+a^{-2} a\right)^{-1}\left(a a^{-1}+a^{-2} a\right)$. Thus,

$$
\begin{aligned}
a a^{-1} & =a a^{-1}\left(a a^{-1}+a^{-2} a\right)^{-1}\left(a a^{-1}+a^{-2} a\right) \\
& =a a^{-1}\left(a a^{-1}+a^{-2} a\right)^{-1}\left(a a^{-1}+a^{-2} a\right) a a^{-1} \\
& =a a^{-1}\left(a a^{-1}+a^{-2} a\right)^{-1}\left(a a^{-1} a+a^{-2} a^{2}\right) a^{-1},
\end{aligned}
$$

that is,

$$
a a^{-1}=a a^{-1}\left(a a^{-1}+a^{-2} a\right)^{-1}\left(a+a^{-2} a^{2}\right) a^{-1} .
$$

On the other hand, from $a \leq_{+} e$, it follows that $a+a^{-2} a^{2} \leq_{+} e+a^{-2} a^{2}$. Now, by (ii), $\left(a^{-2} a^{2}\right)^{2}=a^{-2} a^{2} a^{-1} a^{-1} a^{2}=a^{-2} a a^{-1} a^{2}=a^{-2} a^{2}$, and $e+a^{-2} a^{2} \in E(S)$, and, by (ii) again, we have that

$$
a+a^{-2} a^{2}=\left(a+a^{-2} a^{2}\right)^{2}\left(a+a^{-2} a^{2}\right)^{-1} .
$$

Using (4.2) and (4.3)

$$
\begin{aligned}
a a^{-1} & =a a^{-1}\left(a a^{-1}+a^{-2} a\right)^{-1}\left(a+a^{-2} a^{2}\right) a^{-1} \\
& =a a^{-1}\left(a a^{-1}+a^{-2} a\right)^{-1}\left(a+a^{-2} a^{2}\right)^{2}\left(a+a^{-2} a^{2}\right)^{-1} a^{-1} \\
& =a a^{-1}\left(a a^{-1}+a^{-2} a\right)^{-1}\left(a a^{-1} a+a^{-2} a^{2}\right)\left(a+a^{-2} a^{2}\right)\left(a+a^{-2} a^{2}\right)^{-1} a^{-1} \\
& =a a^{-1}\left(a a^{-1}+a^{-2} a\right)^{-1}\left(a a^{-1}+a^{-2} a\right) a\left(a+a^{-2} a^{2}\right)\left(a+a^{-2} a^{2}\right)^{-1} a^{-1},
\end{aligned}
$$

that is,

$$
a a^{-1}=a a^{-1}\left(a a^{-1}+a^{-2} a\right)^{-1}\left(a a^{-1}+a^{-2} a\right)\left(a^{2}+a a^{-2} a^{2}\right)\left(a+a^{-2} a^{2}\right)^{-1} a^{-1} .
$$

From $a \leq_{+} e$, it follows that $a^{2} \leq_{+} a e \leq_{+} e$. By this, (i) and (ii),

$$
a^{2}=a^{2} a^{-2} a^{2}=a a a^{-2} a^{2} \leq_{+} a e a^{-2} a^{2}=a a^{-1} a e a^{-2} a^{2}=a a^{-2} a^{2},
$$

that is, we have $a^{2}+a a^{-2} a^{2}=a a^{-2} a^{2}$, and if we put this in (4.4),

$$
a a^{-1}=a a^{-1}\left(a a^{-1}+a^{-2} a\right)^{-1}\left(a a^{-1}+a^{-2} a\right) a a^{-2} a^{2}\left(a+a^{-2} a^{2}\right)^{-1} a^{-1} .
$$

It is easy to check that $a^{-1}\left(a a^{-1}+a^{-2} a\right)^{-1}\left(a a^{-1}+a^{-2} a\right) a \leq_{\bullet} a^{-1} a$, which, further, by left amenability of $\leq_{+}$, implies

$$
a^{-1}\left(a a^{-1}+a^{-2} a\right)^{-1}\left(a a^{-1}+a^{-2} a\right) a \leq_{+} a^{-1} a .
$$


This and (4.5) give

$$
\begin{aligned}
a a^{-1} & =a\left(a^{-1}\left(a a^{-1}+a^{-2} a\right)^{-1}\left(a a^{-1}+a^{-2} a\right) a\right) a^{-2} a^{2}\left(a+a^{-2} a^{2}\right)^{-1} a^{-1} \\
& \leq_{+} a\left(a^{-1} a\right) a^{-2} a^{2}\left(a+a^{-2} a^{2}\right)^{-1} a^{-1} \\
& =a a^{-2} a^{2}\left(a+a^{-2} a^{2}\right)^{-1} a^{-1}
\end{aligned}
$$

and

$$
a a^{-1} \leq_{+} a a^{-2} a^{2}\left(a+a^{-2} a^{2}\right)^{-1} a^{-1} .
$$

Now $a^{-2} a^{2} \leq_{+} a+a^{-2} a^{2}$ and (i) imply $a^{-2} a^{2}\left(a+a^{-2} a^{2}\right)^{-1} \leq_{+} a^{-2} a^{2}$. If we put this in (4.6) and use (ii), then

$$
\begin{aligned}
a a^{-1} & \leq_{+} a a^{-2} a^{2}\left(a+a^{-2} a^{2}\right)^{-1} a^{-1} \\
& \leq_{+} a a^{-2} a^{2} a^{-1} \\
& =a a^{-1} a^{-1} a a a^{-1} \\
& =a a^{-1} \cdot a^{-1} a .
\end{aligned}
$$

By Proposition 3.2 it follows that $a a^{-1} \leq_{\bullet} a a^{-1} \cdot a^{-1} a$, and, since $a a^{-1} \cdot a^{-1} a \leq_{\bullet} a a^{-1}$ is evidently true, we have $a a^{-1}=a a^{-1} \cdot a^{-1} a$, that is, $a a^{-1} \leq_{\bullet} a^{-1} a$. Therefore, $a a^{-1}=$ $a^{-1} a$.

The first main result of this section is the following theorem.

THEOREM 4.4. Let $S$ be an inverse semiring. Then $\leq_{+}$is left amenable on $S$ if and only if it is right amenable.

Proof. Let $\leq_{+}$be left amenable on $S$. As $a \leq_{+} a+b$ for any $a, b \in S$, then $a(a+$ $b)^{-1} \leq_{+}(a+b)(a+b)^{-1}$. Let us denote $(a+b)(a+b)^{-1}=e$. Then

$$
a(a+b)^{-1} e=a(a+b)^{-1}(a+b)(a+b)^{-1}=a(a+b)^{-1} .
$$

This implies that

$$
\left(a(a+b)^{-1} e\right)^{-1}=e\left(a(a+b)^{-1}\right)^{-1}=\left(a(a+b)^{-1}\right)^{-1} .
$$

Now, from $a(a+b)^{-1} \leq_{+} e$, and by Theorem 4.3 ,

$$
a(a+b)^{-1}\left(a(a+b)^{-1}\right)^{-1}=\left(a(a+b)^{-1}\right)^{-1} a(a+b)^{-1},
$$

which, by (4.7) and (4.8), gives that

$$
\begin{aligned}
e a(a+b)^{-1} & =e a(a+b)^{-1}\left(a(a+b)^{-1}\right)^{-1} a(a+b)^{-1} \\
& =e\left(a(a+b)^{-1}\right)^{-1} a(a+b)^{-1} a(a+b)^{-1} \\
& =\left(a(a+b)^{-1}\right)^{-1} a(a+b)^{-1} a(a+b)^{-1} \\
& =a(a+b)^{-1}\left(a(a+b)^{-1}\right)^{-1} a(a+b)^{-1} \\
& =a(a+b)^{-1},
\end{aligned}
$$


that is, $a(a+b)^{-1}=(a+b)(a+b)^{-1} a(a+b)^{-1}$. So

$$
a(a+b)^{-1}(a+b)=(a+b)(a+b)^{-1} a(a+b)^{-1}(a+b) .
$$

By left amenability of $\leq_{+}$and $a \leq_{+} a+b$, we have that $a^{-1} a \leq_{\bullet}(a+b)^{-1}(a+b)$, which, further, implies $a^{-1} a=a^{-1} a(a+b)^{-1}(a+b)$. Pre-multiplying this by $a$, we obtain $a=a(a+b)^{-1}(a+b)$, which together with (4.9), gives $a=(a+b)(a+b)^{-1} a$, that is, $a \in(a+b) S$. Thus, by the dual of Theorem $4.2, \leq_{+}$is right amenable.

In a similar manner we can prove the converse.

Theorem 4.2, its dual and Theorem 4.4 give the following corollary.

Corollary 4.5. Let $S$ be an inverse semiring. Then, the following conditions are equivalent:

(i) S satisfies

$$
(\forall a, b \in S) \quad a=a(a+b)^{-1}(a+b) ;
$$

(ii) S satisfies

$$
(\forall a, b \in S) \quad a \in(a+b) S ;
$$

(iii) S satisfies

$$
(\forall a, b \in S) \quad a \in S(a+b) ;
$$

(iv) $E(S)$ is a distributive lattice and

$$
(\forall a, b \in S) \quad(a+b)^{-1}(a+b)=a^{-1} a+b^{-1} b ;
$$

(v) $E(S)$ is a distributive lattice and

$$
(\forall a, b \in S) \quad(a+b)(a+b)^{-1}=a a^{-1}+b b^{-1} ;
$$

(vi) $\leq_{+}$is left amenable;

(vii) $\leq_{+}$is right amenable;

(viii) $\leq_{+}$is amenable.

One of the main results of this paper is the next theorem which gives a solution of the Zeleznikow problem of regular semirings with $\leq_{+}$(left) amenable.

THEOREM 4.6. Let $S$ be a regular semiring with $\leq_{+}$amenable. Then the following conditions are equivalent:

(i) $S_{\bullet}$ is an orthodox semigroup;

(ii) $S_{\bullet}$ is a union of groups (that is, completely regular);

(iii) $S$. is an inverse semigroup;

(iv) $S$. is a Clifford semigroup.

Proof. (i) $\Rightarrow$ (iii). Let $S$ be an orthodox semiring with $\leq_{+}$amenable. By [2, Theorem 13.10], $S$. is a generalized inverse semigroup. This implies that $E(S)$ is a normal band, that is, $E(S)$ satisfies the additional identity $x y z w \approx x z y w$. 
By the assumption and $e \leq_{+} e+f$, there are $e^{*} \in V(e)_{\bullet}$ and $(e+f)^{*} \in V(e+f)$. such that $e^{*} e \leq_{\bullet}(e+f)^{*}(e+f)$. So we get $(e+f) e^{*} e \leq_{\bullet}(e+f)(e+f)^{*}(e+f)=e+f$. By [11, Theorem 6.2.1(iii)] we have $e^{*} \in E(S)$, which, further, implies

$$
\begin{aligned}
(e+f) e^{*} e & =e e^{*} e+f e^{*} e \\
& =e+f e^{*} e e \\
& =e+f e e^{*} e \quad(E(S) \text { is a normal band }) \\
& =e+f e .
\end{aligned}
$$

We now have that $e+f e \leq_{\bullet} e+f$, and, by Lemma 4.1, $e+f e \leq_{+} e+f$. In a similar manner, starting from $f \leq_{+} e+f$ we can prove that $f+e f \leq_{+} e+f$. Thus

$$
\begin{aligned}
(e+f)^{2} & =(e+f e)+(f+e f) \\
& \leq_{+}(e+f)+(e+f) \\
& =e+f .
\end{aligned}
$$

As $e+f \leq_{+}(e+f)^{2}$ is obvious, we have just proved $e+f=(e+f)^{2} \in E(S)$. Once again, from $e \leq_{+} e+f$ and by Lemma 4.1, we have $e \leq_{\bullet} e+f$, that is, we have $e=e(e+f)=e+e f$ and $e=(e+f) e=e+f e$. Hence $e f \leq_{+} e$ and $f e \leq_{+} e$. By Lemma 4.1 again, we have $e f \leq_{\bullet} e$ and $f e \leq_{\bullet} e$. Thus, we have that $e f=e f e$ and $f e=e f e$ which implies $e f=f e$. So, $E(S)$ is a semilattice. It follows from [17, Theorem II.1.2] that $S$ • is an inverse semigroup.

(ii) $\Rightarrow$ (iii). Let $S \bullet$ be a union of groups. Let $\mathcal{L}^{\bullet}\left(\mathcal{R}^{\bullet}\right)$ be Green's $\mathcal{L}(\mathcal{R})$ relation on $S_{\text {. }}$. Suppose that $e, f, g \in E(S)$ are such that $e \leq_{\bullet} g, f \leq_{\bullet} g$ and $e \mathcal{L}^{\bullet} f$. Then we have that $e g=g e=e, f g=g f=f$, ef $=e$ and $f e=f$. On the other hand, by the left amenability of $\leq_{+}$, it follows that $e \leq_{+} g, f \leq_{+} g$, that is, $e+g=g$ and $f+g=g$. Then

$$
\begin{aligned}
& f=g f=(e+g) f=e f+f g=e+f, \\
& e=g e=(f+g) e=f e+g e=f+e .
\end{aligned}
$$

Thus, $e=f$, and $S \bullet$ satisfies $\mathcal{L}^{\bullet}$-majorization. Similarly, we can prove that $S \bullet$ satisfies $\mathcal{R}^{\bullet}$-majorization. Now, by [18, Corollary II.4.12], the natural partial order $\leq_{\bullet}$ is compatible with multiplication.

Let $a \in S$ and $a=a x a=a y a$ for some $x, y \in S$. Since $\leq$. is compatible with multiplication, it means that, by [2, Exercise 13.4], $E(S) \cap x a S y a$ is a semilattice. On the other hand, as $a x=x a$ and $a y=y a$,

$$
x a=x \cdot a y a=x a \cdot y a y a \in E(S) \cap x a S y a
$$

and, similarly,

$$
y a=a y=a x a \cdot y=x a \cdot a y=x \cdot a x a \cdot y a \in E(S) \cap x a S y a .
$$

Thus, $x a \cdot y a=y a \cdot x a$, that is, $x a=y a$ and

$$
x a x=y a x=y a y,
$$

and, by [17, Exercise II.1.13(iii)], $S \bullet$ is an inverse semigroup. 
(iii) $\Rightarrow$ (iv). By the assumption and Theorem 4.2, we have that $\left(a+a^{-1} a\right)^{-1}(a+$ $\left.a^{-1} a\right)=a^{-1} a, a \in S$. This implies, by [17, Lemma II.1.7], that $a \mathcal{L}^{\bullet} a+a^{-1} a$ which, together with $a \leq_{+} a+a^{-1} a$, by [12, Lemma 2.1], gives $\left(a+a^{-1} a\right)\left(a+a^{-1} a\right)^{-1} \leq_{+}$ $a a^{-1}$. Now, by Corollary 4.5, it follows that $\left(a+a^{-1} a\right)\left(a+a^{-1} a\right)^{-1}=a a^{-1}+a^{-1} a$. Therefore, we have that $a^{-1} a \leq_{+} a a^{-1}+a^{-1} a \leq_{+} a a^{-1}$.

On the other hand, by the assumption and Corollary 4.5, we have that $(a+$ $\left.a a^{-1}\right)\left(a+a a^{-1}\right)^{-1}=a a^{-1}, a \in S$, which, further, by [17, Lemma II.1.7], implies that $a \mathcal{R}^{\bullet} a+a a^{-1}$. This and $a \leq_{+} a+a a^{-1}$, by the dual form of [12, Lemma 2.1], give $\left(a+a a^{-1}\right)^{-1}\left(a+a a^{-1}\right) \leq_{+} a^{-1} a$. Once again, by Corollary 4.5, we have that $(a+$ $\left.a a^{-1}\right)^{-1}\left(a+a a^{-1}\right)=a^{-1} a+a a^{-1}$. Thus, we have that $a a^{-1} \leq_{+} a^{-1} a+a a^{-1} \leq_{+} a^{-1} a$. Finally, we get $a a^{-1}=a^{-1} a$, and, $S \bullet$ is Clifford.

(iv) $\Rightarrow$ (i), (iv) $\Rightarrow$ (ii). These are obvious.

As a consequence of Theorems 4.6 and 3.3 we have the following corollary.

Corollary 4.7. An idempotent semiring $B$ is a distributive lattice if and only if $\leq_{+}=\leq$. on $B$.

At the end of this paper we will consider Clifford semirings with $\leq_{+}$amenable. Criteria for a Clifford semiring that guarantee amenability will be given.

THEOREM 4.8. Let $S$ be a Clifford semiring satisfying $\leq_{\bullet} \subseteq \leq_{+}$. Then $\leq_{+}$is amenable if and only if the Clifford semiring $G^{1}$ cannot be embedded into $S$.

Proof. Assume that $\leq_{+}$is not amenable. Then there exist, at least, $a, b \in S$ such that $a \leq_{+} b$, but not $a^{0} \leq_{\bullet} b^{0}$. From $a \leq_{+} b$ it follows that $a^{0} \leq_{+} b a^{-1}$. Let us assume that $b a^{-1} \in E(S)$. Then, by [17, Theorem II.1.4], we have $b a^{-1}=b^{0} a^{0}$, so $a^{0} \leq_{+} b^{0} a^{0}$. Now, by Proposition 3.2, $a^{0} \leq_{\bullet} b^{0} a^{0}$ follows, that is, $a^{0}=b^{0} a^{0}$. Since $b^{0} a^{0} \leq \bullet a^{0}$ is always true, we get $a^{0} \leq . b^{0}$ which is a contradiction. Hence, $b a^{-1} \neq b^{0} a^{0}$.

Using Proposition 3.2 again, we have that

$$
b^{0} a^{0} \leq_{+} a^{0} \leq_{+} b a^{-1} .
$$

Pre-multiplying this by $b a^{-1}$, we have $a^{0} \leq_{+} b a^{-1} \leq_{+}\left(b a^{-1}\right)^{2}$, which implies $\left(b a^{-1}\right)^{m-1} \leq_{+}\left(b a^{-1}\right)^{m}$ for any $m \in Z^{+}$. Let $C=\left\langle b a^{-1}\right\rangle$ be a cyclic subgroup of $H_{b^{0} a^{0}}^{\bullet}$ generated by $b a^{-1}$ with $\left(b a^{-1}\right)^{0}=b^{0} a^{0}$ as the identity (of $C$ ). If $C$ is of finite order, then there exists $n \in Z^{+}$such that $\left(b a^{-1}\right)^{n}=b^{0} a^{0}$. Then,

$$
b^{0} a^{0} \leq_{+} b a^{-1} \leq_{+}\left(b a^{-1}\right)^{2} \leq_{+} \cdots \leq_{+}\left(b a^{-1}\right)^{n-1} \leq_{+}\left(b a^{-1}\right)^{n}=b^{0} a^{0},
$$

that is, we have $b^{0} a^{0}=b a^{-1}$, which is a contradiction. So $C=\left\langle b a^{-1}\right\rangle$ is of infinite order.

Pre-multiplying $b^{0} a^{0} \leq_{+} b a^{-1}$ by $\left(b a^{-1}\right)^{-1}$, we have $\left(b a^{-1}\right)^{-1} \leq_{+} b^{0} a^{0} \leq_{+} a^{0}$. Moreover, we have $\left(\left(b a^{-1}\right)^{-1}\right)^{-m} \leq_{+}\left(\left(b a^{-1}\right)^{-1}\right)^{-(m-1)} \leq_{+} b^{0} a^{0}$ for any $m \in Z^{+}$. Thus

$$
\cdots \leq_{+}\left(b a^{-1}\right)^{-2} \leq_{+}\left(b a^{-1}\right)^{-1} \leq_{+} b^{0} a^{0} \leq_{+} a^{0} \leq_{+} b a^{-1} \leq_{+}\left(b a^{-1}\right)^{2} \leq_{+} \cdots .
$$


So $C^{1}=\left\langle b a^{-1}\right\rangle \cup\left\{a^{0}\right\}$ is an infinite chain under $\leq_{+}$. Hence $C_{+}^{1}$ is a semilattice. As we also have

$$
(\forall s, t \in Z)\left\{\begin{array}{l}
\left(b a^{-1}\right)^{s} \cdot\left(b a^{-1}\right)^{t}=\left(b a^{-1}\right)^{s+t}=\left(b a^{-1}\right)^{t} \cdot\left(b a^{-1}\right)^{s} \\
\left(b a^{-1}\right)^{s} \cdot a^{0}=a^{0} \cdot\left(b a^{-1}\right)^{s}=\left(b a^{-1}\right)^{s}
\end{array}\right.
$$

then $C_{\bullet}^{1}$ is a Clifford semigroup. Thus we have that $\left(C^{1},+, \cdot\right)$ is a Clifford subsemiring of $S$ in which $E\left(C^{1}\right)=\left\{a^{0}, b^{0} a^{0}\right\}$.

We can define a mapping $\varphi: C^{1} \longrightarrow G^{1}$ by

$$
\left\{\begin{array}{l}
\varphi\left(\left(b a^{-1}\right)^{m}\right)=a^{m}, \quad(\forall m \in Z) \\
\varphi\left(a^{0}\right)=1
\end{array}\right.
$$

This is a routine way to verify that $\varphi$ is an isomorphism from the semiring $C^{1}$ onto the Clifford semiring $G^{1}$.

If the Clifford semiring $G^{1}$ can be embedded into $S$, then it is obvious that $\leq_{+}$is not amenable.

\section{References}

[1] T. S. Blyth, 'Dubreil-Jacotin inverse semigroups', Proc. Roy. Soc. Edinburgh (A) 71 (1973), 345-360.

[2] T. S. Blyth, Lattices and Ordered Algebraic Structures (Springer, London, 2005).

[3] T. S. Blyth and M. H. Almeida Santos, 'Amenable orders on orthodox semigroups', J. Algebra 169 (1994), 49-70.

[4] T. S. Blyth and M. H. Almeida Santos, 'Amenable orders associated with inverse transversals', J. Algebra 240 (2001), 143-169.

[5] T. S. Blyth and M. H. Almeida Santos, 'On amenable orders and inverse transversals', Comm. in Algebra (6) 39 (2011), 2189-2209.

[6] N. Chaptal, 'Anneaux dont le demi-groupe multiplicatif est inverse', C. R. Acad. Sci. Paris Ser. A-B 262 (1966), 274-277.

[7] K. Głazek, A Guide to the Literature on Semirings and Their Applications in Mathematics and Information Sciences (Kluwer, Dordrecht, 2002).

[8] J. S. Golan, Semirings and Their Applications (Kluwer, Dordrecht, 1999).

[9] J. S. Golan, Power Algebras over Semirings (Kluwer, Dordrecht, 1999).

[10] J. S. Golan, Semirings and Affine Equations over Them (Kluwer, Dordrecht, 2003).

[11] J. M. Howie, Fundamentals of Semigroup Theory (Oxford University Press, Oxford, 1995).

[12] D. B. McAlister, 'Amenably ordered inverse semigroup', J. Algebra 65 (1980), 118-146.

[13] D. B. McAlister and T. S. Blyth, 'Split orthodox semigroups', J. Algebra 51 (1978), 491-525.

[14] R. McFadden, 'Proper Dubreil-Jacotin inverse semigroups', Glasg. Math. J. 16 (1975), 40-51.

[15] N. R. McKenzie and A. Romanowska, 'Varieties of --distributive bisemilattices, contributions of general algebra', in: Proc. Klagenfurt Conference (Verlag Johannes Hegn, Klagenfurt, 1978), 213-218.

[16] F. Pastijn and X. Z. Zhao, 'Green's $\mathcal{D}$-relation for the multiplicative reduct of an idempotent semiring', Arch. Math. 36 (2000), 77-93.

[17] M. Petrich, Inverse Semigroups (Wiley, New York, 1984).

[18] M. Petrich and N. R. Reilly, Completely Regular Semigroups (Wiley, New York, 1995).

[19] M. K. Sen, Y. Q. Guo and K. P. Shum, 'A class of idempotent semirings', Semigroup Forum 60 (2000), 351-367. 
[20] M. K. Sen and S. K. Maity, 'A note on orthodox additive inverse semirings', Acta Univ. Palack. Olomuc., Fac. Rerum Natur. Math. (1) 43 (2004), 149-154.

[21] H. S. Vandiver, 'Note on simple type of algebra in which cancellation law of addition does not hold', Bull. Amer. Math. Soc. 40 (1934), 914-920.

[22] J. Zeleznikow, 'Orthodox semirings and rings', J. Aust. Math. Soc. (Ser. A) 30 (1980), 50-54.

[23] J. Zeleznikow, 'Regular semirings', Semigroup Forum 23 (1981), 119-136.

YONG SHAO, Department of Mathematics, Northwest University of China, 1 Xuefu Road Changan, 710127 Xi'an, PR China

e-mail: yongshaomath@gmail.com

SINIŠA CRVENKOVIĆ, Department of Mathematics and Informatics, University of Novi Sad, 14 D. Obradovića, 21000 Novi Sad, Serbia e-mail: sima@eunet.rs

MELANIJA MITROVIĆ, Faculty of Mechanical Engineering, University of Niš, 14 A. Medevedeva, 18000 Niš, Serbia e-mail: meli@masfak.ni.ac.rs 